\title{
Fractional-order sliding mode controller for the two-link robot arm
}

\author{
Trong-Thang Nguyen \\ Faculty of Electrical and Electronic Engineering, Thuyloi University, Vietnam
}

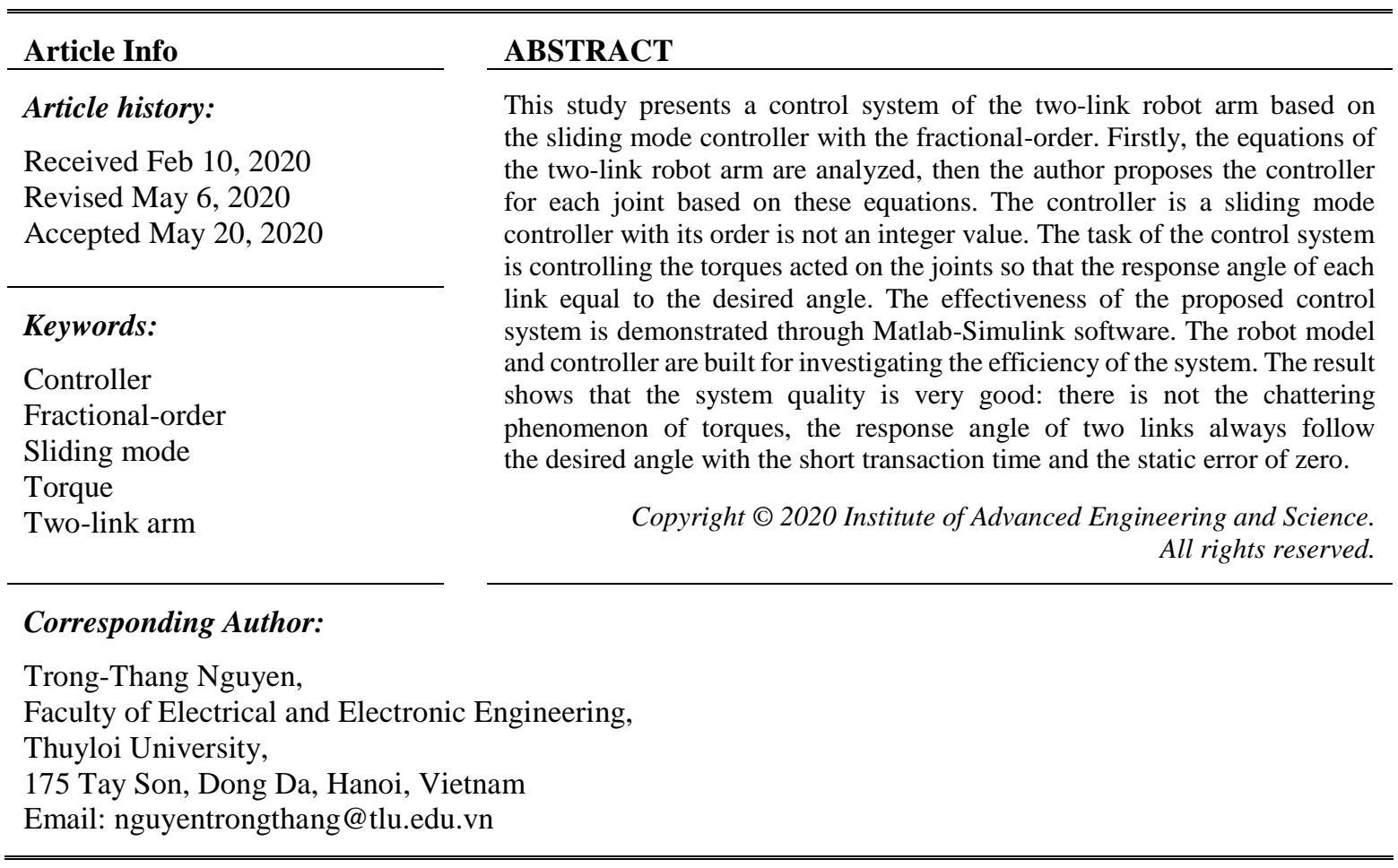

\section{INTRODUCTION}

Robots have been applied for industrial production in the 1950s with the role of replacement of human labor, improving the product quality and the efficiency of production. There are common missions, which the robot address very well, such as manufacturing, material handling, and painting, etc. Recently, robots are more and more playing an important role in life and industrial production. Therefore, it is very important to develop the performance of the robot application. There is some research on robot control [1-3], the controller that is most used for the robot is the proportional-integral-derivative (PID) one. However, because of the nonlinear characteristic of the robot, so the PID controller is not suitable, the effectiveness is low.

There are some nonlinear methods for robot control, for example the linearization technique [4], the back-stepping control method [5], etc. However, these methods have the drawback, i.e., the control signal has the chattering. A number of approaches have been contributed to robot control, which includes adaptive control [6], optimal control [7], robust control [8], and intelligent control [9, 10]. Whereas, these mentioned methods endure some shortcomings that is the complexity of the computation. In order to minimize this limitation, fuzzy control, which is considered an efficient and simple solution, was utilized based on the experiences of a human [11, 12] but its sustainability is quite low. With the intention of enhancing sustainability, the fuzzy control associated with the PID controller was presented [13, 14], however, the deficiency of this proposal is that the output response is not quick.

The study [15] introduced a sliding mode controller to solve the above constraints. In recent years, the sliding mode controller, which is powerful in controlling both linear and nonlinear objects, has been examined by various researchers. Emelyanov and his co-workers first introduced this method in the early 1950s. This controller offers some advantages involving the quick output response, the robustness and stability, the simple control algorithm, and the good transient performances. However, the limitation of the sliding mode 
controller is the high frequency chattering of the control signals, which leads to undesirable loads on control actuators $[16,17]$. In order to diminish the above cons, the author presents the control system for the two-link robot arm using the fractional-order sliding mode control.

The fractional-order sliding mode controller is a sliding mode controller where the order of sliding surface is a fractional value. The fractional-order sliding mode controller has many advantages compared to the traditional sliding mode controller. It can even control the objects with uncertain dynamical model [18]. Hence, recently, the fractional-order sliding mode controller has been investigated and applied into many system controls such as: single-link flexible manipulator [19], antilock braking systems [20, 21], speed control system for permanent magnet synchronous motor [22]. As a result, the systems with the fractional-order sliding mode controller are of superior quality to traditional controllers. Because of the above advantages, in this paper, based on the dynamic model of the two-link robot arm, the author will build a suitable fractional-order sliding mode controller and prove the stability of the whole system through the theory of Lyapunov. The achieved findings will be transparently displayed through Matlab Simulink. The results will indicate that there is not a high-frequency chattering in the control signals as well as a static error, the quality of the control system is adequate, and the response angle of two links quickly approaches the desired angle.

The remains of the paper are as follows: Section 2 presents the calculus of derivatives and integrals of fractional order, the kinematic equation of two-link robot arm, and the fractional-order sliding mode controller. Section 3 presents the results and analysis. Finally, The conclusions are presented in section 4.

\section{DESIGNING THE CONTROLLER}

\subsection{The calculus of derivatives and integrals of fractional order}

Leibniz and L'Hopital propose fractional calculus on the basis of the integer-order calculus in 1695 [23]. The first-order derivative is determined by the (1):

$$
f^{\prime}(t)=\lim _{h \rightarrow 0} \frac{f(t)-f(t-h)}{h}
$$

Therefore, the second-order and n-order derivative are determined as follows:

$$
\begin{aligned}
& f^{\prime \prime}(t)=\lim _{h \rightarrow 0} \frac{f^{\prime}(t)-f^{\prime}(t-h)}{h}=\lim _{h \rightarrow 0} \frac{f(t)-2 f(t-h)+f(t+2 h)}{h^{2}} \\
& f^{(3)}(t)=\lim _{h \rightarrow 0} \frac{f^{\prime \prime}(t)-f^{\prime \prime}(t-h)}{h}=\lim _{h \rightarrow 0} \frac{f(t)-3 f(t-h)+3 f(t-2 h)-f(t-3 h)}{h^{3}} \\
& f^{(n)}(t)=\lim _{h \rightarrow 0} \frac{\sum_{i=0}^{n}(-1)^{i}\left(\begin{array}{l}
n \\
i
\end{array}\right) f(t-i . h)}{h^{n}}
\end{aligned}
$$

where:

$$
\left(\begin{array}{l}
n \\
i
\end{array}\right)=\frac{n !}{i !(n-i) !}
$$

In (5), $n$ is a natural number. If $n=z$ is not a natural number, (5) is rewritten as follows:

$$
\left(\begin{array}{l}
Z \\
i
\end{array}\right)=\frac{\Gamma(z+1)}{\Gamma(i+1) \Gamma(z-i+1)}
$$

where $\Gamma($.$) is the gamma function.$

For generalizing, Riemann-Liouville and Caputo have defined the fractional order for the calculus of derivatives and integrals as follows [24]:

$$
f^{(z)}=\frac{1}{\Gamma(n-z)} \int_{0}^{t} \frac{f^{(n)}(\tau)}{(t-\tau)^{z+n-1}} d \tau
$$

where: $\Gamma(z)=\int_{0}^{\infty} t^{z-1} e^{-t} d t, n-1<z<n$ ( $\mathrm{n}$ is an integer value). If $z<0$ then the calculus (7) is integration, else if $z>0$ then the calculus (7) is differentiation. Especially, if $z=1$ the calculus (7) is first order derivative function. 


\subsection{The model of the two-link robot arm}

Figure 1 illustrates the two-link robot arm model, in which $m_{1}$ and $l_{1}$ represent Link1's mass and length; $m_{2}, l_{2}$ stand for Link2's mass and length; T1, T2 represent the torque of Link1 and Link2; $\theta_{1}$ and $\theta_{2}$ represent the Link1 angle and Link2 angle.

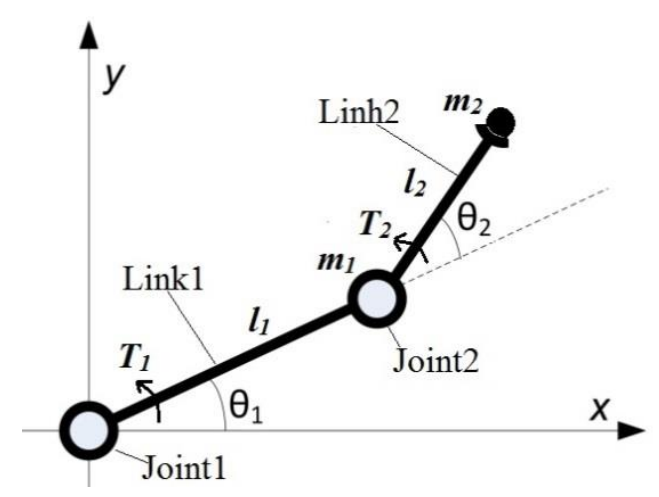

Figure1. The model of two-link robot arm

The dynamic equation of the two-link robot arm is as the following $[25,26]$ :

$$
\left[\begin{array}{l}
T_{1} \\
T_{2}
\end{array}\right]=\left[\begin{array}{ll}
D_{11} & D_{12} \\
D_{21} & D_{22}
\end{array}\right]\left[\begin{array}{l}
\theta_{1} \\
\theta_{2}
\end{array}\right]+\left[\begin{array}{l}
C_{1} \\
C_{2}
\end{array}\right]\left[\begin{array}{l}
\theta_{1} \\
\theta_{2}
\end{array}\right]+\left[\begin{array}{l}
G_{1} \\
G_{2}
\end{array}\right]
$$

where:

$$
\begin{gathered}
D_{11}=\left(m_{1}+m_{2}\right) l_{1}^{2}+m_{2} l_{2}^{2}+2 m_{2} l_{1} l_{2} \cos \left(\theta_{2}\right) \\
D_{12}=D_{21}=m_{2} l_{2}^{2}+m_{2} l_{1} l_{2} \cos \left(\theta_{2}\right) \\
D_{22}=m_{2} l_{2}^{2} \\
G_{1}=\left(m_{1}+m_{2}\right) g l_{1} \cos \left(\theta_{1}\right)+m_{2} g l_{2} \cos \left(\theta_{1}+\theta_{2}\right) \\
G_{2}=m_{2} g l_{2} \cos \left(\theta_{1}+\theta_{2}\right) \\
C_{1}=-m_{2} l_{1} l_{2} \sin \left(\theta_{2}\right) \dot{\theta}_{2}-m_{2} l_{1} l_{2} \sin \left(\theta_{2}\right)\left(\dot{\theta}_{1}+\dot{\theta}_{2}\right) \\
C_{2}=m_{2} l_{1} l_{2} \sin \left(\theta_{2}\right) \dot{\theta}_{1}
\end{gathered}
$$

Rewriting (8), we get:

$$
\tau=D \cdot \ddot{\theta}+C \cdot \dot{\theta}+G
$$

where:

$$
\tau=\left[\begin{array}{l}
T_{2} \\
T_{2}
\end{array}\right] ; D=\left[\begin{array}{ll}
D_{11} & D_{12} \\
D_{21} & D_{22}
\end{array}\right] ; \ddot{\theta}=\left[\begin{array}{l}
\ddot{\theta}_{1} \\
\ddot{\theta}_{2}
\end{array}\right] ; C=\left[\begin{array}{l}
C_{1} \\
C_{2}
\end{array}\right] ; \dot{\theta}=\left[\begin{array}{c}
\dot{\theta}_{1} \\
\dot{\theta}_{2}
\end{array}\right] ; \quad G=\left[\begin{array}{c}
G_{1} \\
G_{2}
\end{array}\right]
$$

2.3. The control system of two-link robot arm based on the fractional-order sliding mode controller Setting the fractional-order sliding surface as follows:

$$
s=\lambda_{1} \cdot e+\lambda_{2} \cdot e^{(-\alpha)}+\lambda_{3} \cdot e^{\beta}+\dot{e}
$$

where $e$ is the error:

$\theta_{\text {set }}=\left[\begin{array}{l}\theta_{1} \text { set } \\ \theta_{2 \_s e t}\end{array}\right]$ is the vector of desired angle values;

$$
e=\theta_{\text {set }}-\theta
$$

$\theta=\left[\begin{array}{l}\theta_{1} \\ \theta_{2}\end{array}\right]$ is the vector of response angle values;

$\alpha, \beta$ are fractional oders, those values are positive real values. 
$\lambda_{1}, \lambda_{2}, \lambda_{3}$ are vectors of positive gain parameters.

The differential of the (10) is as:

$$
\dot{s}=\lambda_{1} \cdot \dot{e}+\lambda_{2} \cdot e^{(1-\alpha)}+\lambda_{3} \cdot e^{(1+\beta)}+\ddot{e}
$$

Setting the control signal $u=\left[\begin{array}{l}T_{1} \\ T_{2}\end{array}\right]$ so that $\dot{s}=-K . \operatorname{sign}(s)(K>0)$. The torque force is:

$$
\tau=D \cdot\left(\lambda_{1} \cdot \dot{e}+\lambda_{2} \cdot e^{(1-\alpha)}+\lambda_{3} \cdot e^{(1+\beta)}+K \cdot \operatorname{sign}(s)\right)+C \cdot \dot{\theta}+G
$$

Choosing Lyapunov function $V=\frac{1}{2} s^{2}$.

If the control signal is set up according to the equation (12), $\dot{s}=-K$. $\operatorname{sign}(s)$. We have:

$$
\dot{V}=s . \dot{s}=-K .|s|<0 \forall s
$$

Thus, the system will attain the asymptotical stability at the equilibrium point $s=0$, which indicates that $e$ and all derivative of $e$ will equal zero.

\section{THE RESULTS AND ANALYSIS}

According to the dynamic (8) and the model introduced in Figure 1, the model of the two-link robot arm is constructed on Matlab-Simulink and presented in Figure 2. Parameters of robot are set up as the followings: $l_{l}=1(\mathrm{~m}) ; l_{2}=0.6(\mathrm{~m}) ; m_{l}=0.7(\mathrm{~kg}) ; m_{2}=0.4(\mathrm{~kg})$. The primary angles of two links:

$$
\underline{\theta}=\left[\begin{array}{l}
\theta_{1} \\
\theta_{2}
\end{array}\right]=\left[\begin{array}{l}
-\pi / 6 \\
-\pi / 12
\end{array}\right](\mathrm{rad})
$$

Figure 3 presents the diagram of the controller which is constructed following the (12). We set parameters of the controller as below:

$$
\lambda_{1}=\left[\begin{array}{l}
4 \\
4
\end{array}\right] ; \quad \lambda_{2}=\left[\begin{array}{l}
6 \\
6
\end{array}\right] ; \quad \lambda_{3}=\left[\begin{array}{l}
3 \\
3
\end{array}\right] ; \underline{K}=\left[\begin{array}{l}
5 \\
5
\end{array}\right] ; \alpha=0.4 ; \beta=0.6
$$

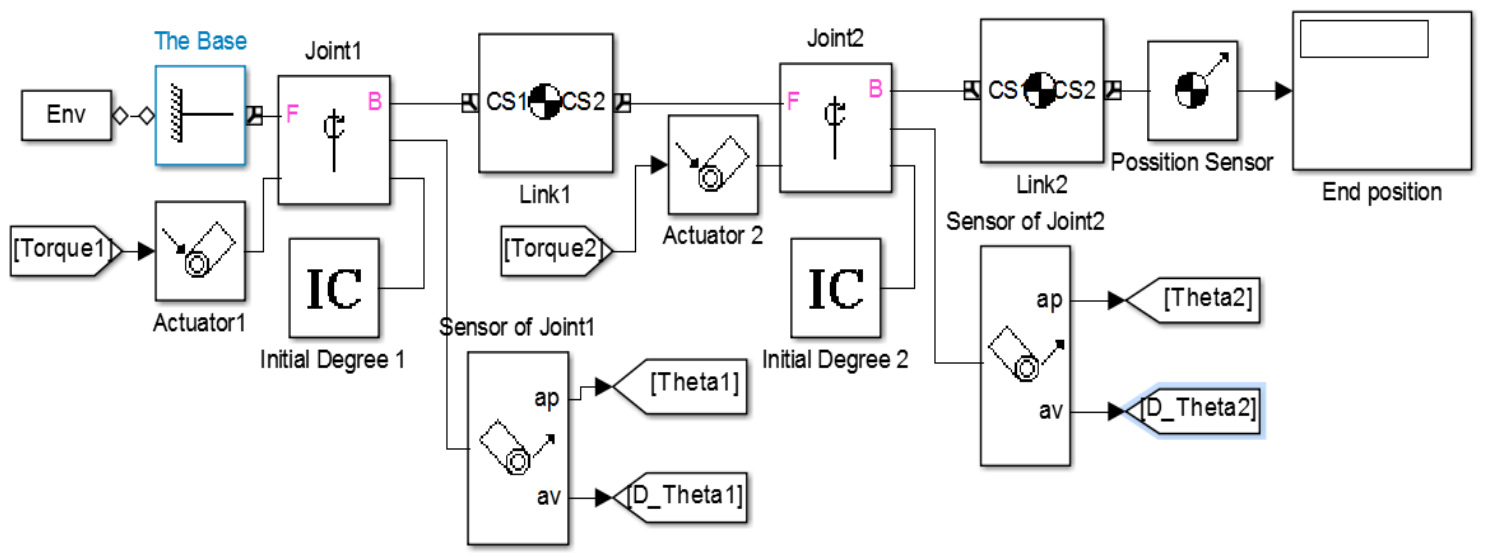

Figure 2. The two-link robot arm model

Running this system, the achieved results are presented in Figures 4-7. The phase-status trajectories of two joints is displayed in Figure 4. The results of simulation indicate that all status trajectories approach the origin $O(0,0)$ without oscillations. Thus, the response angles $(\theta)$ reach the expected angles $\left(\theta_{\text {set }}\right)$. 


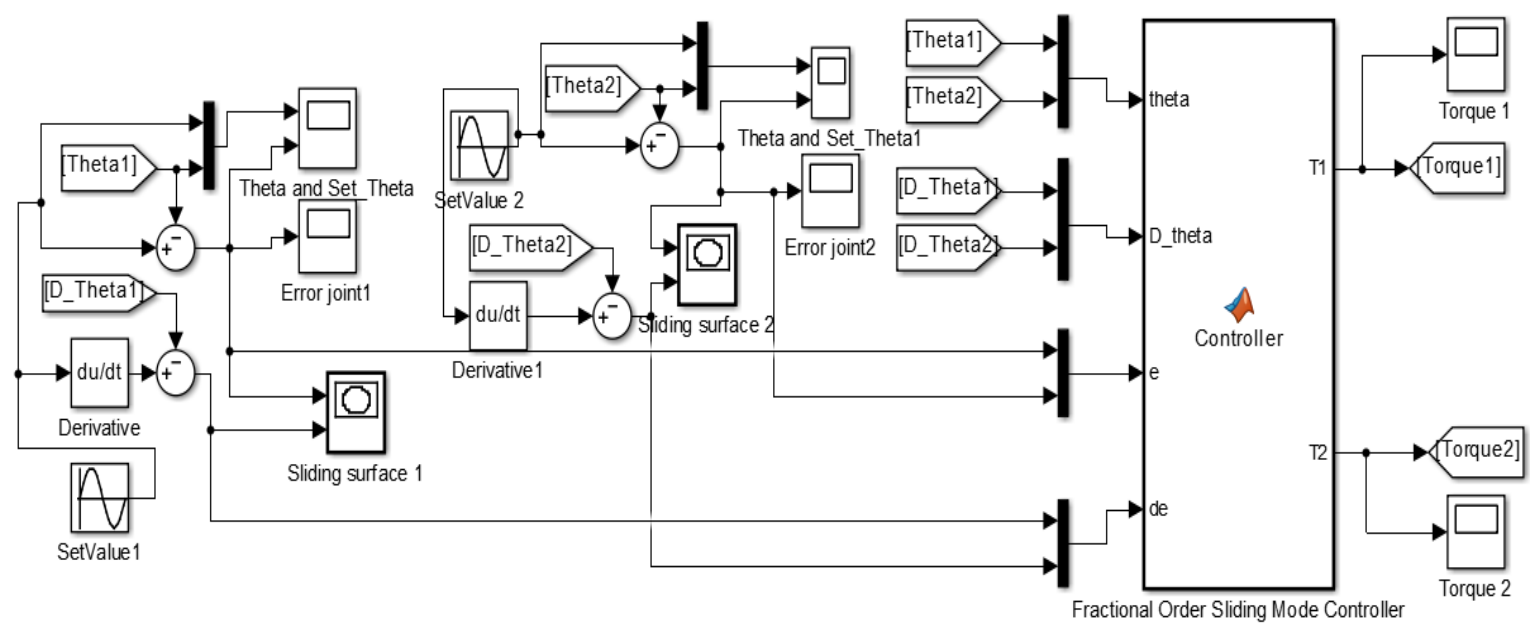

Figure 3. The model of controller

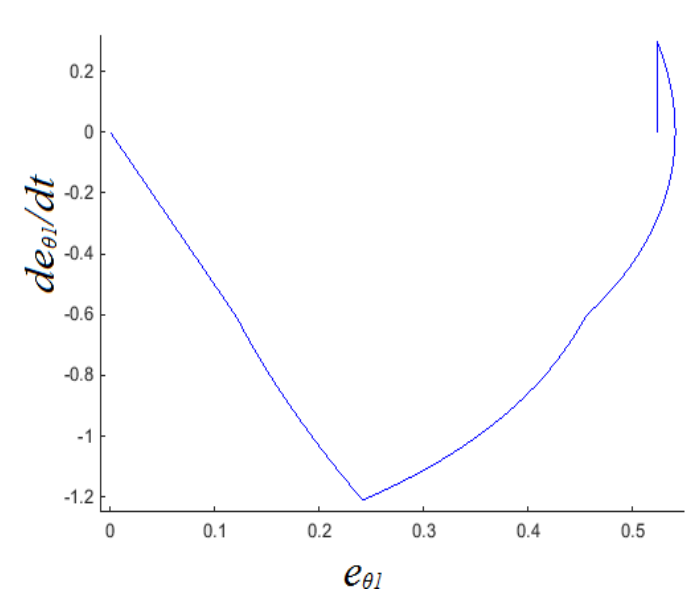

(a)

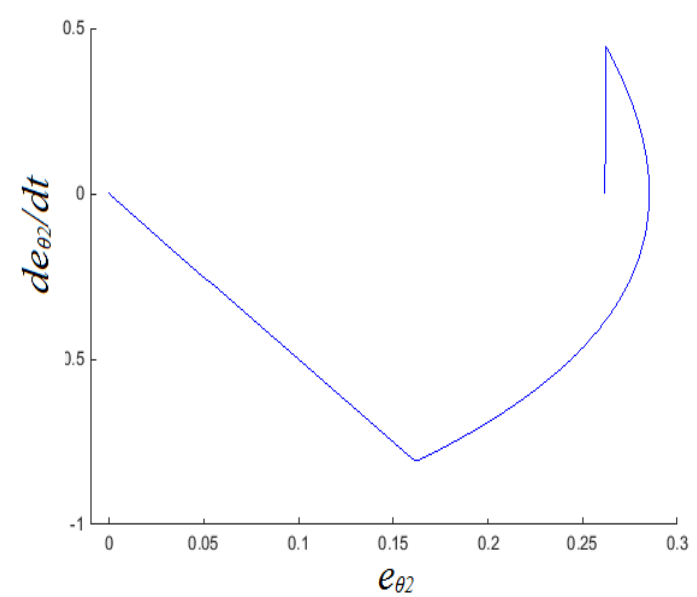

(b)

Figure 4. The phase-status trajectories, (a) the joint1, (b) the joint2
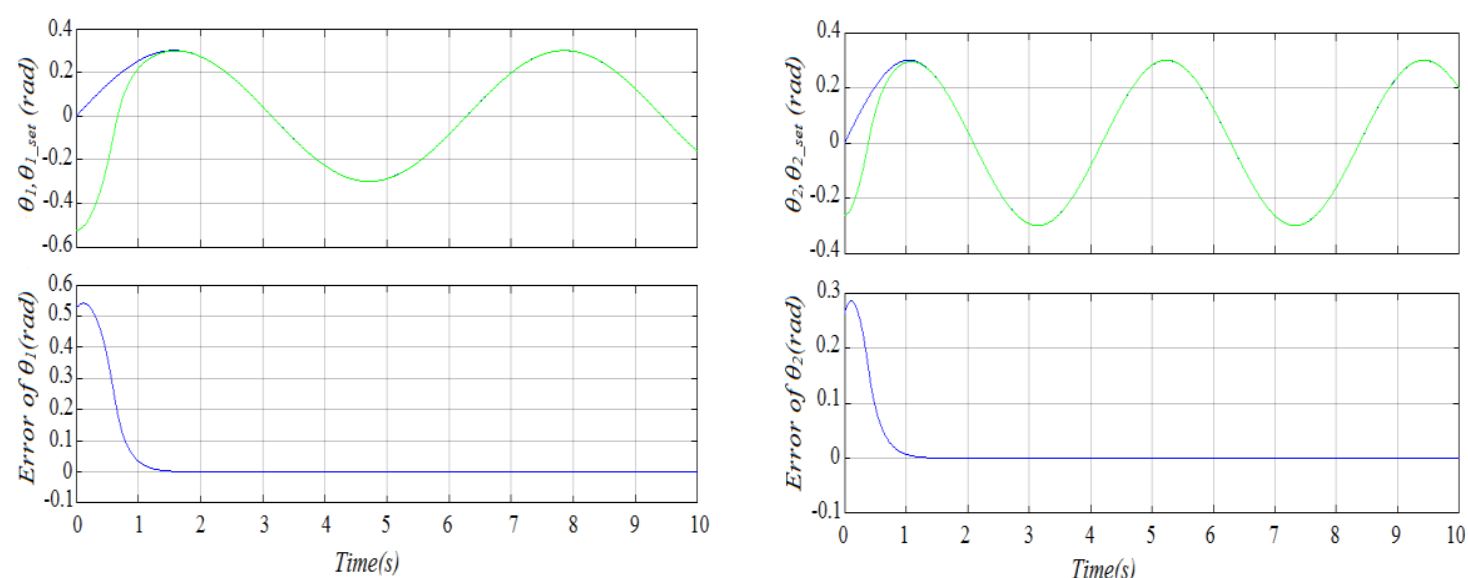

Figure 5. The response angle and control error of the joint 1

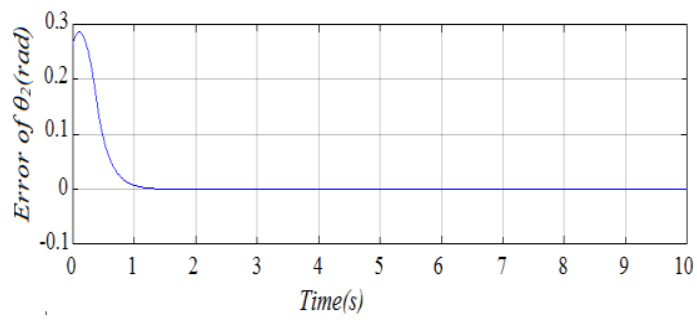

Figure 6. The response angle and control error of the joint 2 
Figures 5 and 6 show the time characteristics of Link1 and Link2 respectively. Each characteristic includes the desired angle $\theta_{\text {set }}$, the response angle $(\theta)$, and the $\operatorname{error}\left(\theta_{\text {set }}-\theta\right)$. The outcomes of simulation demonstrate that: firstly, the response angles are not equal to the expected angles, then the response angles meet the expected values after a short time (approximately 1s), and finally, the response angles always adhere the desired values, the errors $\left(e_{1}, e_{2}\right)$ are zero. Accordingly, it can be asserted that the control system has a high quality. The control torque of each joint is shown in Figure 7. The simulation results show that there is not the chattering phenomenon of torques, which is necessary for the system to assure the sustainability of actuators and devices.

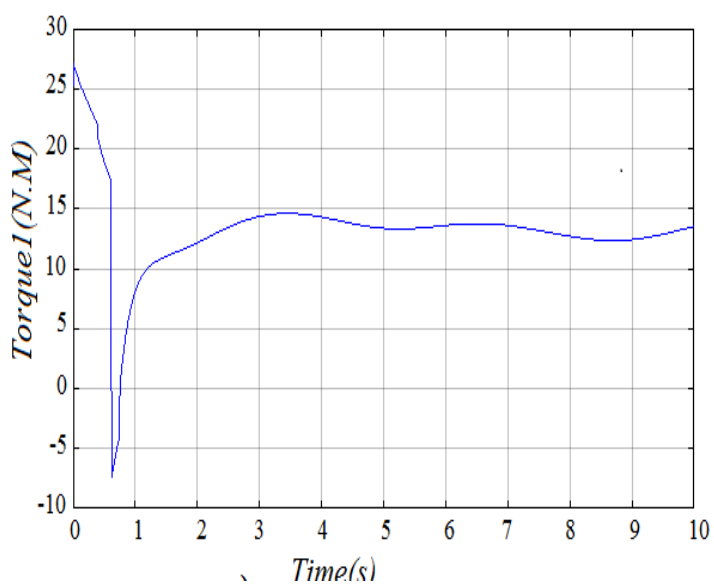

(a)

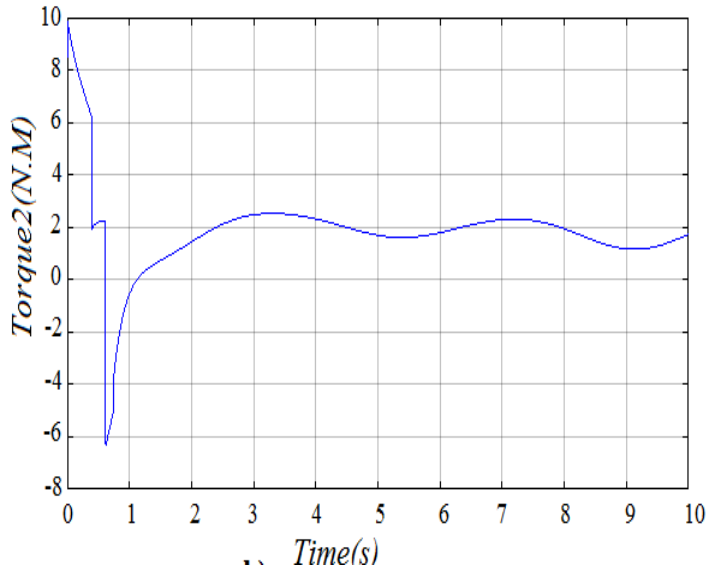

(b)

Figure 7. The control torque of two joints, (a) the joint1, (b) the joint2

\section{CONCLUSION}

In the present research, the author gained success in establishing the control system for the two-link robot arm utilizing the fractional-order sliding mode controller. The simulation outcomes displayed that the control system has remarkably high quality, the response angle of two links always reaches the desired angles in a short duration and the static error equals zero. Notably, the chattering phenomenon does not exist in the torques and therefore the lifespan of the controller and actuator is increased. Since the algorithms of the controller are analyzed specifically, the proposed controller is established quite easily in practice. The success of this proposed algorithm is the basis for the authors to conduct experiments in further studies.

\section{REFERENCES}

[1] I. David and G. Robles, "PID control dynamics of a Robotic arm manipulator with two degrees of Freedom," Control de Processos y Robotica, pp. 1-6, 2012.

[2] P. Rocco, "Stability of PID control for industrial robot arms," IEEE transactions on robotics and automation, vol. 12, no. 4, pp. 606-614, 1996.

[3] J. Y. Dieulot and F. Colas, "Robust PID control of a linear mechanical axis: A case study," Mechatronics, vol. 19 , no. 2 , pp. 269-273, 2009.

[4] F. J. C. Garcia, et al., "On the Design of a 4 Degrees-of-Freedom Pick and Place Cable Suspended Parallel Manipulator," IAES International Journal of Robotics and Automation (IJRA), vol. 6, no. 4, pp. 286-302, 2017.

[5] M. Muhammad, et al., "Velocity control of a two-wheeled inverted pendulum mobile robot: a fuzzy model-based approach," Bulletin of Electrical Engineering and Informatics, vol. 8, no. 3, pp. 808-817, 2019.

[6] W. Ham, "Adaptive control based on explicit model of robot manipulator," IEEE transactions on automatic control, vol. 38, no. 4, pp. 654-658, 1993.

[7] S. Kumar, et al., "Robotic Arm Movement optimization using Soft Computing," IAES International Journal of Robotics and Automation (IJRA), vol. 6, no. 1, pp. 1-14, 2017.

[8] M. R. Soltanpour, et al., "Robust nonlinear control of robot manipulator with uncertainties in kinematics, dynamics and actuator models," International Journal of Innovative Computing, Information and Control, vol. 8, no. 8, pp. 5487-5498, 2012.

[9] S. Nurmaini and B. Tutuko, "Intelligent Robotics Navigation System: Problems, Methods, and Algorithm," International Journal of Electrical and Computer Engineering (IJECE), vol. 7, no. 6, pp. 3711-3726, 2017. 
[10] R. Koker and A. Ferikoglu, "Model based intelligent control of a 3-joint robotic manipulator: A simulation study using artificial neural networks," in International Symposium on Computer and Information Sciences, pp. 31-40, 2004.

[11] A.S. Handayani, et al., "Analysis on swarm robot coordination using fuzzy logic," Indonesian Journal of Electrical Engineering and Computer Science, vol. 13, no. 1, pp. 48-57, 2019.

[12] G. C. Hwang and S. C. Lin, "A stability approach to fuzzy control design for nonlinear systems," Fuzzy sets and Systems, vol. 48, no. 3, pp. 279-287, 1992.

[13] S. J. Huang and J. S. Lee, "A stable self-organizing fuzzy controller for robotic motion control," IEEE Transactions on Industrial Electronics, vol. 47, no. 2, pp. 421-428, 2000.

[14] J. L. Meza, et al., "Fuzzy Self-Tuning PID Semiglobal Regulator for Robot Manipulators," IEEE Transaction Industrial Electronics, vol. 59, no. 6, pp. 2709-2717, 2012.

[15] Y. Ihedrane, et al., "Improved wind system using non-linear power control," Indonesian Journal of Electrical Engineering and Computer Science, vol. 14, no. 3, pp. 1148-1158, 2019.

[16] L. M. Fridman, “An averaging approach to chattering," IEEE Transactions on Automatic Control, vol. 46, no. 8, pp. 1260-1265, 2001.

[17] J. J. E. Slotine and W. Li., “Applied Nonlinear Control,” Prentice Hall Inc., London, UK, 1991.

[18] S. Dadras and H. R. Momeni, "Fractional terminal sliding mode control design for a class of dynamical systems with uncertainty," Communications in Nonlinear Science and Numerical Simulation, vol. 17, no. 1, pp. 367-377, 2012.

[19] A. Mujumdar, et al., "Fractional order sliding mode control for single link flexible manipulator," in 2013 IEEE International Conference on Control Applications (CCA), pp. 288-293, 2013.

[20] Y. Tang, et al., "Fractional order sliding mode controller design for antilock braking systems," Neurocomputing, vol. 111, pp. 122-130, 2013.

[21] Y. Tang, et al., "Adaptive fuzzy fractional-order sliding mode controller design for antilock braking systems," Journal of Dynamic Systems, Measurement, and Control, vol. 138, no. 4, pp. 1-8, 2016.

[22] B. Zhang, et al., "Fractional order sliding-mode control based on parameters auto-tuning for velocity control of permanent magnet synchronous motor," ISA transactions, vol. 51, no. 5, pp. 649-656, 2012.

[23] Y. Q. Chen, et al., "Fractional order control-a tutorial," in 2009 American control conference, pp. 1397-1411, 2009.

[24] N. A. Camacho, et al., "Lyapunov functions for fractional order systems," Communications in Nonlinear Science and Numerical Simulation, vol. 19, no. 9, pp. 2951-2957, 2014.

[25] T. N. Trong and M. N. Duc, "Sliding Surface in Consensus Problem of Multi-Agent Rigid Manipulators with Neural Network Controller," Energies, vol. 10, no. 12, pp. 2127-2140, 2017.

[26] R. M. Murray, et al., "A mathematical introduction to robotic manipulation,” CRC press, 1994. 